\title{
Effect of Surface Wettability on the Droplet Impact Morphologies on Dry Smooth Polycarbonate Surfaces
}

\author{
Patrick Foltyn*1, Daniela Ribeiro², André Silva², Grazia Lamanna ${ }^{1}$, Bernhard Weigand ${ }^{1}$ \\ ${ }^{1}$ University of Stuttgart, Institute for Aerospace Thermodynamics (ITLR), Stuttgart, Germany \\ ${ }^{2}$ University of Beira Interior, AEROG-LAETA, Covilhã, Portugal \\ *Corresponding author email: patrick.foltyn@itlr.uni-stuttgart.de
}

\begin{abstract}
The surface wettability has a significant influence on the morphology and spreading behavior during droplet impacts on dry smooth walls. On the way for predicting spreading diameters in dependency of the wetting behavior, the experimental database was extended by an experimental study in which distilled water and isopropanol droplets have impacted onto dry smooth Lexan ${ }^{\circledR}$ (Polycarbonate) surfaces at four different impact velocities. The range of Reynolds numbers was set between 1,135 and 12,240 and for the Weber number between 80 and 1,165. The surface material, and thus also the characteristic surface roughness, were kept constant, while the wetting behavior was modified using plasma activation and plasma polymerization processes. Different contact angles have been investigated in a range from full wetting to nonwetting for water and from full wetting to partial wetting for isopropanol. The experiments have been conducted on a newly designed test rig. High-speed diffuse backlight images at $20 \mathrm{kHz}$ from the top and the lateral perspective are acquired on a shared CMOS-sensor simultaneously. A bottom perspective in a total internal reflection configuration is acquiring the footprint of the droplet impact. This enables to better define the maximum spreading diameter and to distinguish between wetted and non-wetted areas.
\end{abstract}

\section{Keywords}

Wetting behavior; apparent contact angle; droplet spreading; high-speed imaging; plasma processes

\section{Introduction}

The investigation of droplet impacts on surfaces has been performed by many scientists for over a hundred years, mainly starting with the well-known publications of the researchers Worthington, Clifton, Edgerton and Killian [1, 2, 3]. But still, the physical processes during the droplet impacts are not fully understood and with scientific and industrial progress new fields of research are opened. Droplet impacts on walls can be found in almost all parts of everyday life: soil erosion during heavy rains, ink-jet printing, combustion engines, spray coating, spray cooling, just to name some applications [4].

Several researchers have investigated the droplet impact on dry solid surfaces and the influence of impact energy, surface roughness, and wettability on the physical outcome, which can be deposition, prompt splash, crown splash, receding breakup, partial rebound, and complete rebound [5]. The found splashing limits are well-fitting for the respective droplet sizes and the impact conditions used by the researchers, e.g. Bai and Gosman [6], Mundo et al. [7], or Vander Wal et al. [8]. In general, one can state, the higher the impact energy the more likely splashing will occur. This is also amplified by roughness, which means that an increasing roughness can promote splashing.

About the influence of wettability on the spreading rate of droplets, a scientific debate can be found. While Mao et al. [9] have found that the equilibrium contact angle has no significant influence on the maximum spreading diameter, Rioboo et al. [10] and Moita and Moreira [11] could show that with increasing contact angle the tendency of receding break up is strongly 
promoted and the spreading rate can be also increased, respectively. A possible explanation of the unclear influence of wettability can be the findings of Pasandideh et al. [12], which have shown that the significance of the wettability influence is depending on the Weber number.

To analyze the influence of wettability on the droplet spreading behavior, droplet impacts have been investigated using three perspectives (top, lateral, bottom) acquired by synchronized highspeed cameras. The bottom perspective is a total-internal reflection configuration whose working principle was developed more than 20 years ago at the ITLR and is described in [13, 14]. As test fluids, distilled water and isopropanol were used, which were impacting at four different impact velocities on a smooth dry Lexan ${ }^{\circledR}$ (Polycarbonate, PC) surface. The surface wettability could be flexibly adjusted using plasma activation and plasma polymerization. Contrary to the most literature studies, the wettability was always modified for the same surface material. This ensures that the influence of changed materials with possible slight surface roughness changes are avoided. The impact conditions are ranging from 1,135 $<R e<12,240$ and $80<W e<1,165$. Here, the Reynolds and Weber numbers are defined as $R e=\rho D_{0} u_{0} / \mu$ and $W e=\rho u_{0}^{2} D / \gamma$, respectively, using the droplet impact diameter $D_{0}$, the impact velocity $u_{0}$, the droplet fluid density $\rho$ and the surface tension $\gamma$.

\section{Material and methods}

\section{Definition, determination and modification of contact angles}

The wetting ability of liquids can be determined by investigating the contact angle. The latter is defined as the angle on the liquid side between the tangent of the solid-liquid and the liquidvapor interface. The equation of Young [15] describes with the Young contact angle $\theta_{Y}$ the energetic minimum and, therefore, the favorable shape of the droplet. For the determination of the Young contact angle $\theta_{Y}$, the free surface energies $\gamma$ of the solid-vapor (SV), solid-liquid $(\mathrm{SL})$ and the liquid-vapor (LV) interface are used.

$$
\gamma_{S V}-\gamma_{S L}=\gamma_{L V} \cos \theta_{Y}
$$

Due to the fact that surface imperfections, e.g. inhomogeneities of the surface free energies, nanometric roughness, etc., are unavoidable, the physical system will attain a contact angle which can be in the global energy minimum or in a local minimum of the Gibbs energy [16].

For the characterization of the wetting behavior of the used surface sample, this apparent contact angle $\theta_{\text {app }}$ has been determined using an Optical Contour Analysis (OCA) system of DataPhysics Instruments $\mathrm{GmbH}$. With the help of the sessile drop method using an ellipse for fitting the droplet shape, the arithmetic mean of the contact angles on the left and right triple points have been obtained. This angle is then used for classifying the wettability of the surface sample and for further evaluations.

The natural apparent contact angle $\theta_{\text {app }}$ of distilled water and the used Lexan ${ }^{\circledR}$ surface was measured to be $\theta_{\text {app }}=79.8^{\circ}$. For isopropanol, the liquid fully wetted the surface $\left(\theta_{\text {app }} \approx 0^{\circ}\right)$. This apparent contact angle must be controlled to evaluate the influence of wettability on the physical processes during the droplet impact. By varying at least one of the free surface energies in equation (1), the equilibrium contact angle and the wettability can be modified. By plasma activating the surface samples in a non-thermal plasma in air at a pressure of $155 \mathrm{~Pa}$, the free surface energy of the solid can be modified [17]. The process duration controls the magnitude of activation and, consequently, the apparent contact angle for water so that the wetting behavior could be set either to full $\left(\theta_{\mathrm{app}} \approx 0^{\circ}\right)$ or to a partial wetting $\left(\theta_{\mathrm{app}} \in\left[25^{\circ}-40^{\circ}\right)\right.$ ). Full wetting for water is considered if the contact angle is that flat, so it cannot be measured anymore. For isopropanol, no further plasma activation was necessary, due to the already full-wetting properties.

Moreover, plasma polymerization could be used to apply a nanometric coating onto the existing smooth surface samples. For both, the roughness and the optical properties, no alterations are detectable. However, the measurable apparent contact angles for distilled water and isopropanol are increasing to $\theta_{\mathrm{app}} \in\left[117^{\circ}-122^{\circ}\right]$ and $\theta_{\mathrm{app}} \in\left[66^{\circ}-73^{\circ}\right]$, respectively. Therefore, in 
Table 1. Summary of the evaluated parameters. For each column, the indicated parameter range was investigated. The parameters that required a pre-treatment by plasma activation or polymerization are marked.

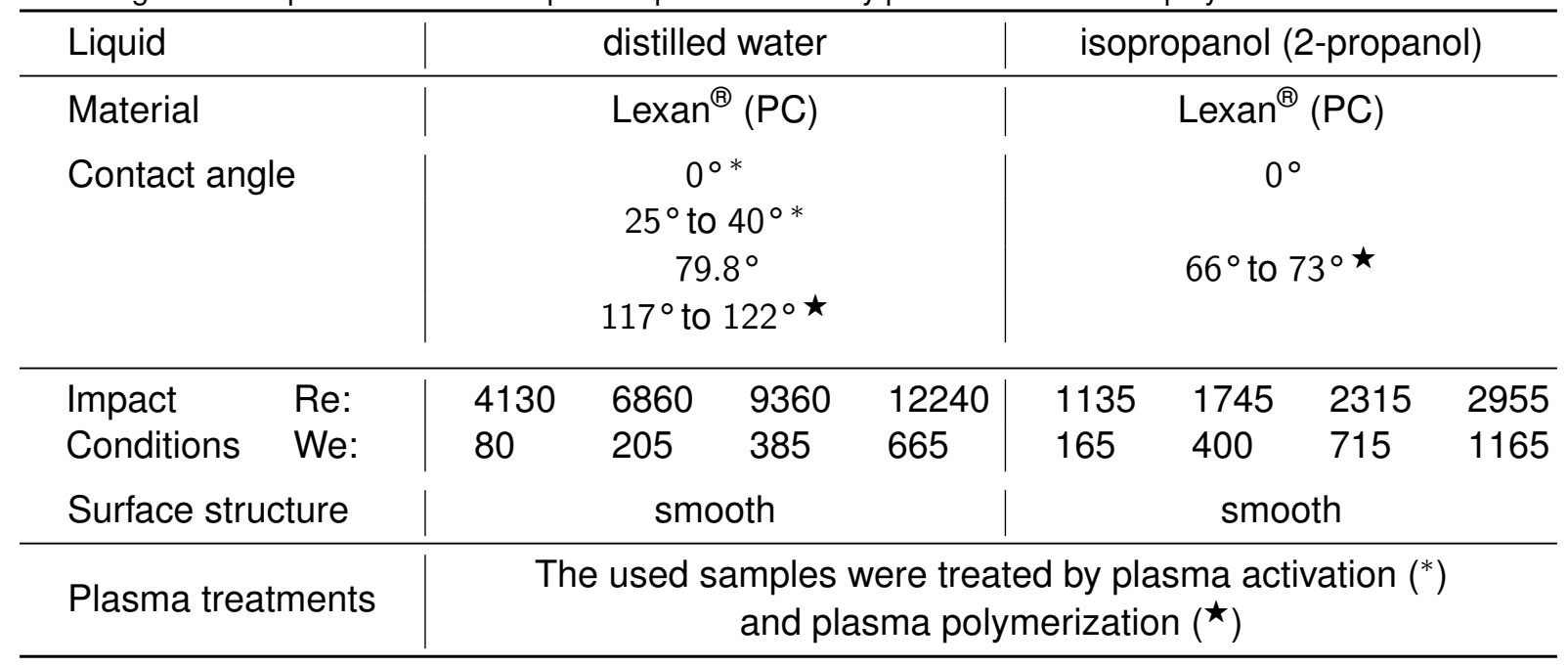

total four different wetting behaviors for water and two different wetting behaviors for isopropanol could be evaluated, see Tab. 1.

\section{Experimental setup}

The experimental setup consists of three main parts: a droplet generation unit, a triggering and synchronization unit as well as an imaging unit.

For the droplet generation, a blunt needle with an outer diameter of $0.4 \mathrm{~mm}$ is used, which was tilted by $45^{\circ}$ in order to ensure a determinable spot of droplet detachment and, therefore, to have a more deterministic droplet trajectory. A syringe pump is constantly feeding the blunt needle with the respective fluid, in order to ensure a homogeneous droplet chain with constant droplet diameters detaching at a frequency of approximately $1.5 \mathrm{~Hz}$. For the droplet impact, one random droplet is selected in passing the droplet barrier to access the test section and impacting onto the polymer surface with specific wetting properties, see red cross in Fig. 1. When the droplet is cutting a LASER sheet, the acquisition of the cameras is triggered using TTL-signals. The droplet impacts have been observed by three different perspectives, i.e. top, lateral and bottom, in order to get as much information of the physical processes as possible. The images have been acquired using two Photron SA-X2 high-speed cameras with a shutter time of $1 / 88,888 \mathrm{~s}$, a frame rate of $20,000 \mathrm{fps}$ and a resolution of $1,024 \times 672 \mathrm{px}^{2}$ each. In order to synchronize all perspectives, first, the top and lateral perspectives have been combined on one CMOS-chip and, second, the cameras have been electronically synchronized. The optical concept of the test rig is shown in Fig. 1.

For the top and lateral perspectives, parallel light is generated, which is then directed by optical elements onto the impact point. To ensure a better light distribution for the lateral perspective, a diffusor plate has been used. Then, the light path is entering two similar Schneider Kreuznach Symmar 80/5.6 objectives for intermediate imaging of each perspective. For the combination of the two perspectives, a 50:50 beam splitter plate is used so that it can be used as mirror for the lateral perspective and as transmitting plate for the top perspective. The light rays are led into a WILD Heerbrugg M8 Zoom stereomicroscope to which a high-speed camera is mounted. The intermediate imaging was necessary to be able to combine both perspectives side-by-side onto one CMOS-chip, because the working distance of the microscope with approximately $9 \mathrm{~cm}$ was too short for the geometrical arrangement. An exemplary image sequence of an impacting droplet seen by the top and lateral perspective is depicted in Fig. 3 .

For the bottom perspective, a total-internal reflection configuration was selected, which is also the reason why a prism is used as main optical element carrying the smooth polymeric surface 


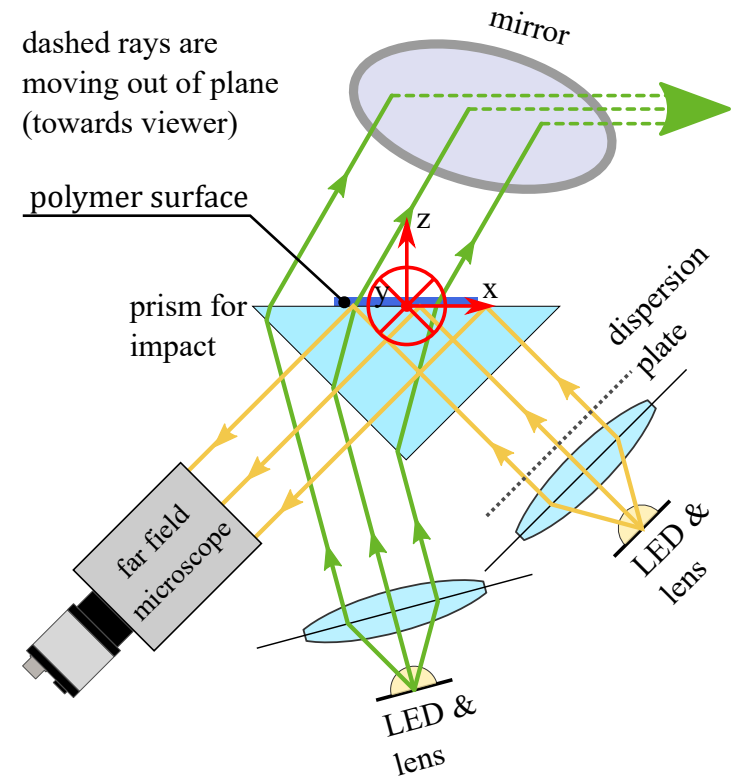

(a)

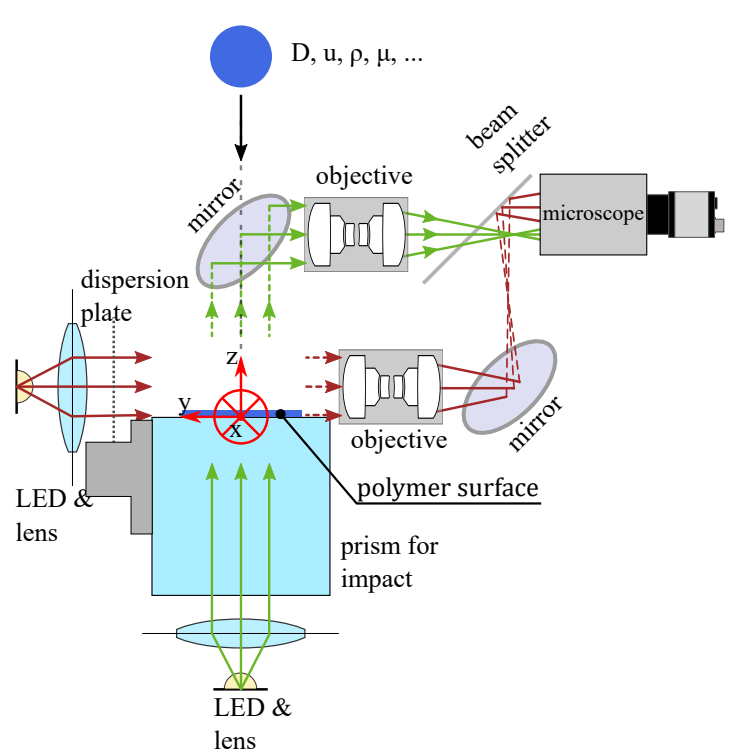

(b)

Figure 1. The optical concept of the droplet impact facility: (a) front view with the concept of the total internal reflection configuration of the bottom perspective, (b) lateral and top perspective (bottom perspective is for sake of simplicity not displayed). The red cross is considered as the droplet impact point on the polymer surface to be investigated and at the same time the origin of the test rig's coordinate system.

samples on which the droplets are impacting. With the help of this perspective, the wetted and non-wetted areas can be easily determined as reported [13,14]. Here, Snell's Law [18] shows that there is a critical angle of incidence $\xi_{\text {crit }}$ for the light rays, measured between the light ray and the interface normal, at which the light is reflected and not refracted.

$$
\xi_{\text {crit }}=\arcsin n_{2} / n_{1}
$$

This angle is dependent on the refractive indices of the adjacent optical layers $n_{2} / n_{1}$. For dry and horizontal surfaces, this relation is fulfilled, so that the light ray is reflected at the upper (horizontal) prism surface. For wetted surfaces, however, two main influences can alter this relation. First, the orientation of the surface is due to the liquid not horizontal anymore, and second, the relation of refractive indices has been changed. In both cases, the light rays are in that particular area refracted instead of reflected and, thus, the camera can obtain darker areas. In case equation (2) is still fulfilled for a wetted surface at the liquid-vapor interface, the height difference due to the film will force the liquid sheet to be tilted at some areas. Therefore, also for that case, a proper assignment of wetted and non-wetted areas can be guaranteed since the wetted areas on the camera sensors are, then, always surrounded by a dark circular area. An exemplary image sequence of an impacting droplet seen by the bottom perspective is depicted in Fig. 3 bottom row.

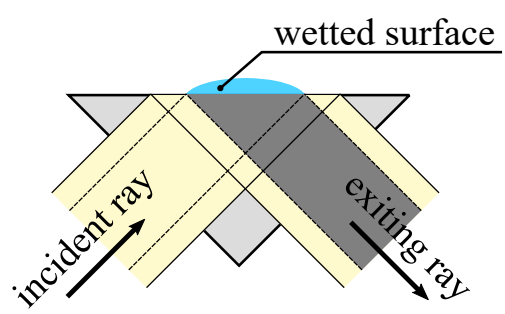

(a)

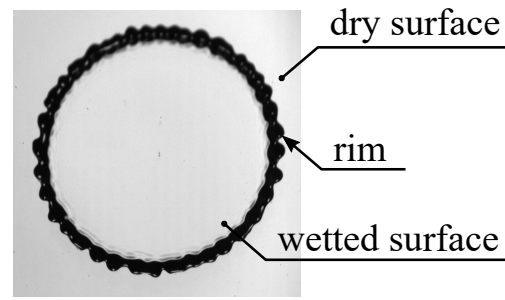

(b)

Figure 2. (a) Principle of the total internal reflection configuration and (b) exemplary image with indicated dry and wetted areas 


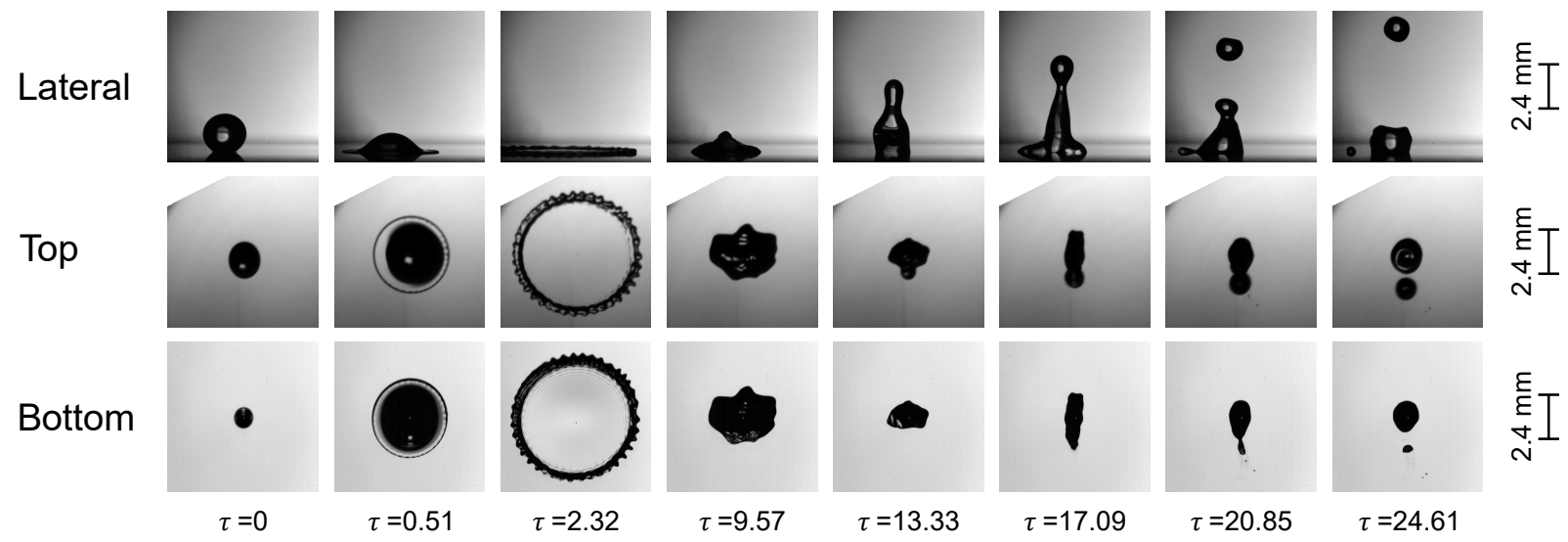

Figure 3. Exemplary sequence of partially rebounding water droplet at a dry smooth surface seen from all three perspectives; $R e=6,860 \mathrm{We}=205$

\section{Parameter Space}

The influence of the wetting behavior on the droplet spreading has been investigated using the parameters summarized in Tab. 1 . In total four different droplet falling heights, $0.15,0.35$, 0.70 , and $1.30 \mathrm{~m}$, have been chosen which have resulted for the two used droplet fluids, distilled water and isopropanol, into a parameter range of $1,135<R e<12,240$ and $80<W e<1,165$. The wettability of the smooth has been modified, as explained before, using plasma activation and plasma polymerization for the indicated cases in Tab. 1. For water four different wetting behaviors and for isopropanol, two different wetting behaviors were evaluated. In general, at least ten experimental repetitions have been performed which showed always the repeatability of the experiments. Therefore, the presented dimensions in the following section are always corresponding to the arithmetic mean values of the obtained dimensions.

\section{Results and discussion}

In Fig. 3, an exemplary image sequence of a partially rebounding droplet is shown for all three perspectives. The dimensionless time $\tau$ is defined as $\tau=t u_{0} / D_{0}$, where $t$ is considered as the time relative to the impact. In the first column $(\tau=0)$, the droplet touches at first the surface. This can be observed very well especially using the bottom perspective. With increasing time, the droplet spreads and a horizontal droplet lamella surrounded by a rim can be observed for $\tau=2.32$, the time of the maximum spreading diameter $\tau\left(D_{\max }^{*}\right)$. After the receding of the droplet lamella a liquid jet is forming and a secondary droplet is ejected.

For the general droplet impact morphologies, droplet splashing can be observed for the highest three falling heights $(R e>1,745, W e>400)$ of isopropanol droplets while for distilled water always droplet deposition was obtained, which is also in accordance with the splashing limit of Vander Wal et al. [8]. For a hygrophilic wetting behavior, meaning $\theta_{\text {app }}<90^{\circ}$ no influence of the wettability on the splashing and deposition outcome was trackable. However, for hygrophobic behavior, partial rebound could be observed for some cases. This coincides with the observations in the literature, in which the wettability has no significant influence for $\theta_{\text {app }}<90^{\circ}$ on the splashing/deposition threshold [19].

A summary of the temporal development of the spreading diameter of droplets impacting from different falling heights in Fig. 4a shows that independently of the wetting behavior and the Weber number, the spreading follows approximately the known proportionality of $D^{*} \sim \tau^{1 / 2}$ during the kinematic and spreading phase [10]. Here $D^{*}$ corresponds to the dimensionless droplet spreading diameter defined as $D^{*}=D / D_{0}$. Depending on the wettability, the droplet continues spreading very slowly (isopropanol, $\theta_{\text {app }} \approx 0^{\circ}$ ) or the receding has various strengths (dist. water, $\theta_{\mathrm{app}} \in\left[117^{\circ} ; 122^{\circ}\right]$ ) leading for some indicated cases even to partial rebound. In the case of full-wetting isopropanol $\left(\theta_{\mathrm{app}} \approx 0^{\circ}\right)$, the maximum spreading diameter is not reached 


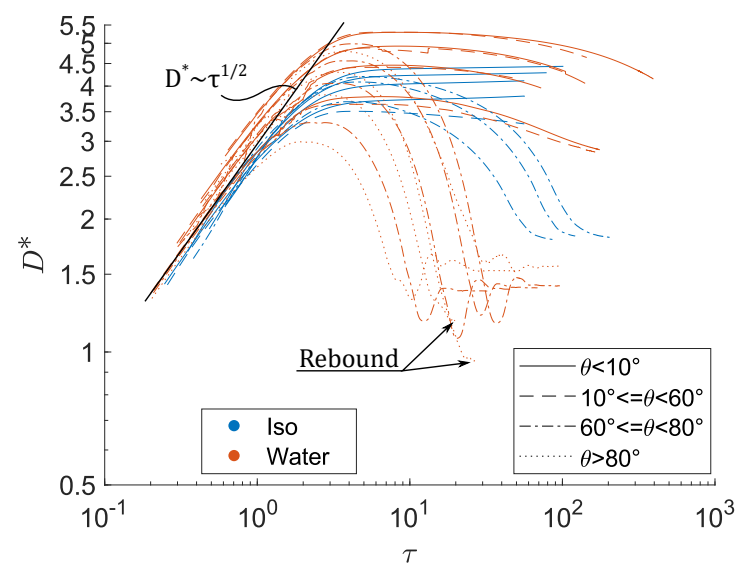

(a)

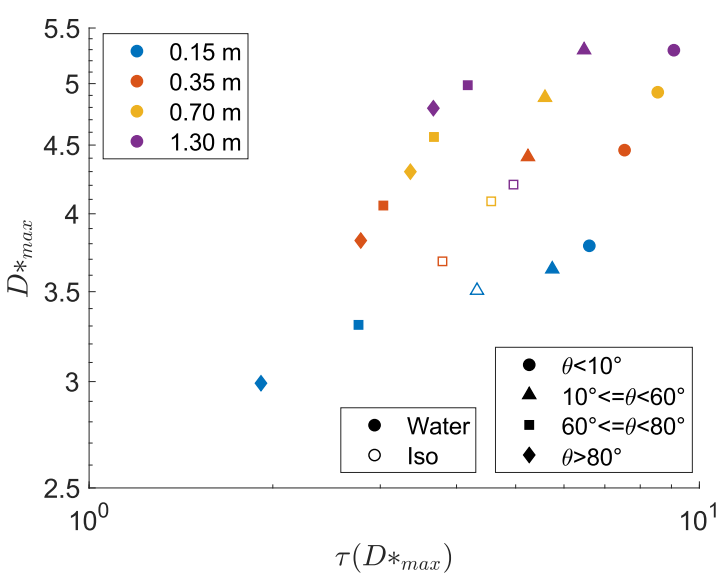

(b)

Figure 4. (a) Dimensionless droplet spreading diameter $D^{*}$ over dimensionless time $\tau$; (b) Maximum spreading diameter $D^{*}$ and the corresponding dimensionless time $\tau$, when this diameter is reached

after the complete kinematic impact energy has been dissipated in the splash. The spreading slowly continues at a rate of $D^{*} \sim \tau^{0.007}$, showing probably a superposition of Tanner's law of spreading [20] and the evaporation of the droplet fluid. In contrast to that, the very slow receding movement of distilled water with $\theta_{\text {app }} \approx 0^{\circ}$ might be explained by a more than three times higher surface tension and an apparent contact angle of $\theta_{\text {app }} \approx 0^{\circ}$ but still $\theta_{\text {app }} \neq 0^{\circ}$.

In the following, the full-wetting isopropanol cases $\left(\theta_{\mathrm{app}} \approx 0^{\circ}\right)$ cannot be used due to the continuous spreading. A comparison of the maximum spreading diameter shows, that the impact energy (We number), the wettability $\left(\theta_{\mathrm{app}}\right)$ and the fluid (e.g. viscosity $\mu$ ) have a significant influence, see Fig. 4b: The higher the impact energy and wettability, the larger the spreading diameter and the longer the spreading occurs until the maximum spreading is reached. In addition, keeping the apparent contact angle approximately constant and increasing the viscosity will decrease the maximum spreading diameter but extend the time slightly at which this diameter is reached. Mostly similar results can be found in [10].

Hence, it appears reasonable to normalize the dimensionless maximum spreading diameter as $\widetilde{D}^{*}{ }_{\max }=D_{\max }^{*} / D_{\max , \theta_{\mathrm{app}}=0^{\circ}}$ in order to assess the reduction of the spreading diameter for increasing contact angles while all other parameters are kept constant. Moreover, the dimen-

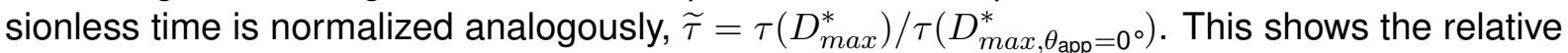
time decrease to reach the maximum spreading diameter for less wettable surfaces in comparison to the full wetting cases.

In Fig. 5a, only the data points of distilled water are used because isopropanol provided only two wetting behaviors. With this approach, it can be clearly demonstrated that for all impact energies, the behavior is almost unique. In agreement to the findings of Moita and Moreira [11], it could be shown that decreasing the wettability will lead to a reduction of the spreading diameter and spreading time. The magnitude of reduction follows for all impact heights the empirically fitted power law

$$
{\widetilde{D^{*}}}_{\max }=-0.00185 \widetilde{\tau}^{-2.07}+1.02
$$

Comparing the normalized maximum spreading diameter in dependency of the surface wettability in Fig. 5b, the influence of the kinetic energy can be clearly shown. The lower the kinetic energy, the higher the relative differences in the spreading diameters for different surface wettabilites. As the impact energies are increasing, the effect of wettability is slowly diminishing, as reported by Pasandideh et al. (1996) [12]. Therefore, it can be expected, that for very fast droplets, as they are found, e.g. in combustion engines, the influence of wettability can be fully neglected. 


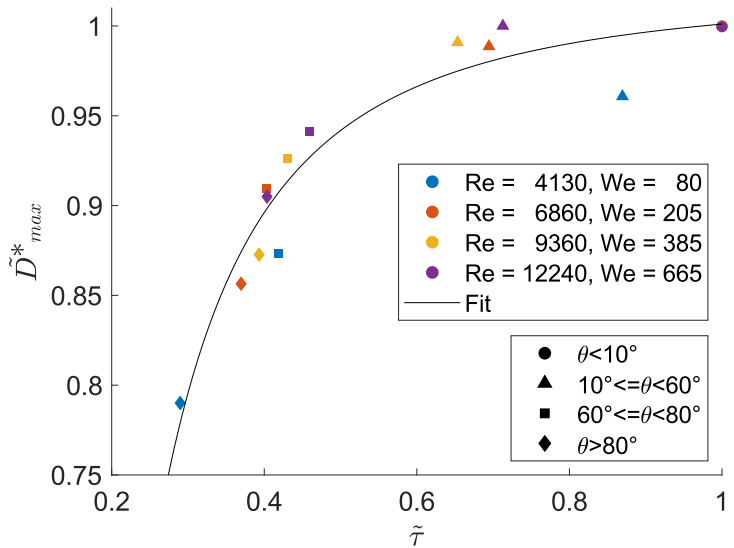

(a)

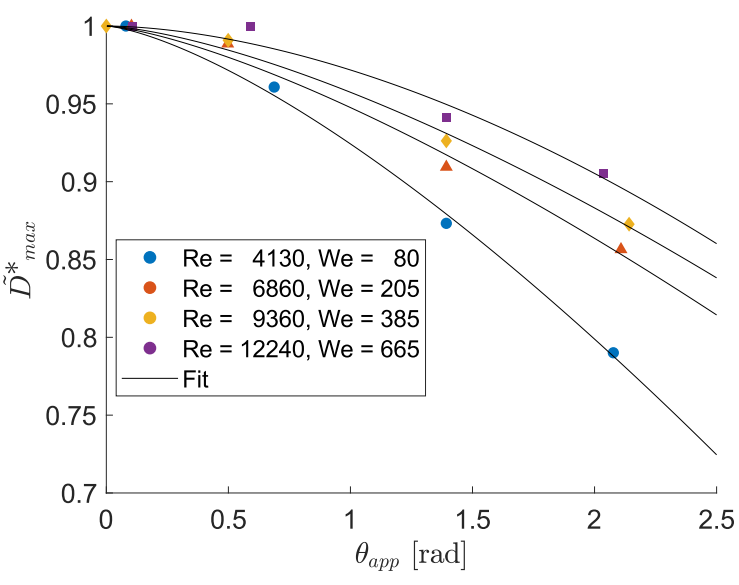

(b)

Figure 5. (a) Normalized maximum $\tilde{D}^{*}$ and the corresponding normalized dimensionless time $\tilde{\tau}$ for distilled water droplet impacts; (b) Normalized maximum spreading diameter of distilled water over the apparent contact angle $\theta_{\text {app }}$ showing the dependency of the diameter rise and the impact energy (falling height)

\section{Conclusions}

An experimental study to investigate the influence of the wetting behavior on the droplet impact and spreading on smooth polymeric Lexan ${ }^{\circledR}$ surfaces has been investigated. For this, an experimental setup using three synchronized perspectives, a top and a lateral using diffuse backlight imaging and a bottom perspective in a total internal reflection configuration, has been introduced. The use of plasma activation and plasma polymerization allowed to modify the wettability for the surface samples, while the material and its characteristic surface roughness could be kept constant over all experiments.

The evaluation of the experimental data has shown that with increasing impact energy and increasing wettability, which has concurrently a lower apparent contact angle $\theta_{\mathrm{app}}$, the maximum spreading diameter is increasing. With increasing viscosity, the maximum spreading diameter is getting smaller while the time at which this diameter is reached is slightly increasing. In normalizing the dimensionless spreading factor and the dimensionless time, the influence of the wettability for a constant impact energy could be identified. While the absolute influence of the wettability is decreasing with increasing impact energy, the relative influence within the same impact energy but variable wetting behavior seems to be identical.

Further work needs to be done using more fluids and surface materials to be able to improve the estimating correlation.

\section{Acknowledgements}

The authors kindly acknowledge the financial support of this work by the Deutsche Forschungsgemeinschaft (DFG) in the frame of the International Research Training Group "Droplet Interaction Technologies" (GRK 2160: DROPIT) under the project number 270852890. Additionally, the authors would like to acknowledge the support of Fundação para a Ciência e a Tecnologia (FCT) through the Ph.D. scholarship SFRH/BD/140009/2018 and the project UIDB/50022/2020.

\section{Nomenclature}
$D, D_{0}$
Diameter, impacting droplet diameter [m]
$D^{*}$
Dimensionless spreading diameter $D^{*}=d / D_{0}[-]$
$n_{1}, n_{2}$
Refractive indices for different media (1 \& 2) [-]
$t$
Time [s] 


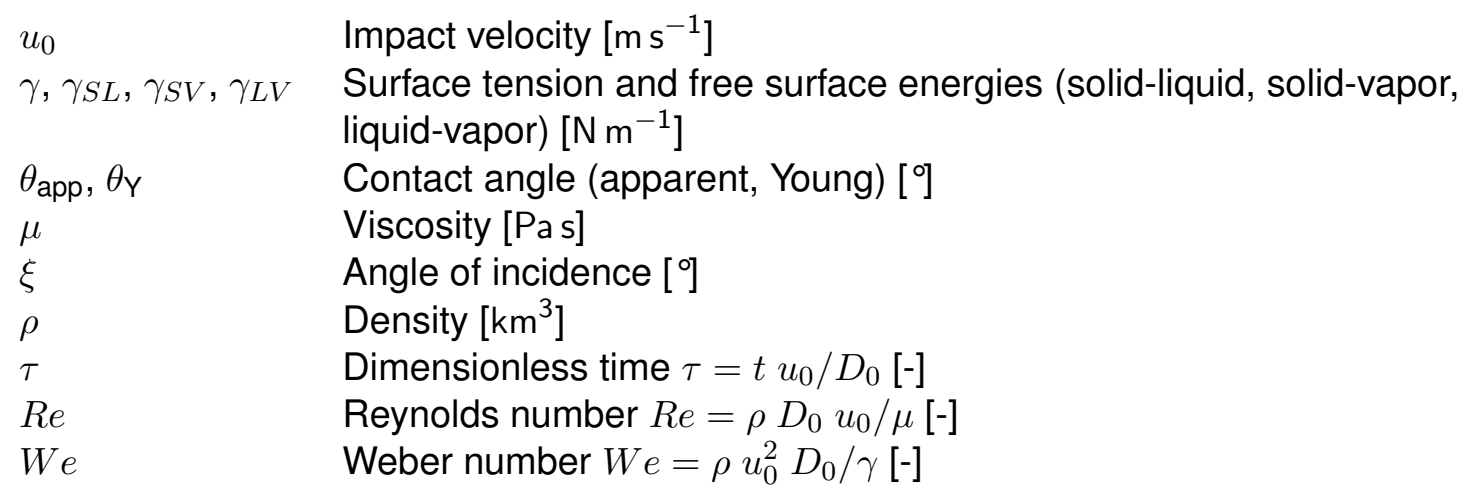

\section{References}

[1] Worthington, A. M., and Clifton, R. B., 1877. Proceedings of the Royal Society of London, 25 (171-178), pp. 261-272.

[2] Worthington, A., 1908. A Study of Splashes. Nineteenth Century Collections Online (NCCO): Photography: The World through the Lens.

[3] Edgerton, H., and Killian, J., 1939. Flash!: Seeing the Unseen by Ultra High-speed Photography.

[4] Yarin, A., Roisman, I., and Tropea, C., 2017. Collision Phenomena in Liquids and Solids.

[5] Rioboo, R., Tropea, C., and Marengo, M., 2001. Atomization and Sprays, 11 (2), pp. 155165.

[6] Bai, C., and Gosman, A. D., 1995. In International Congress \& Exposition, SAE International.

[7] Mundo, C., Sommerfeld, M., and Tropea, C., 1995. International Journal of Multiphase Flow, 21 (2), pp. $151-173$.

[8] Vander Wal, R. L., Berger, G. M., and Mozes, S. D., 2006. Experiments in Fluids, 40 (1), Jan, pp. 53-59.

[9] Mao, T., Kuhn, D. C. S., and H, T., 1997. AlChE Journal, 43 (9), pp. 2169-2179.

[10] Rioboo, R., Marengo, M., and Tropea, C., 2002. Experiments in Fluids, 33 (1), July, pp. 112-124.

[11] Moita, A. S., and Moreira, A. L. N., 2003. In Proceedings in 9th ICLASS - Europe, Institute for Liquid Atomization and Spray Systems.

[12] Pasandideh-Fard, M., Qiao, Y. M., Chandra, S., and Mostaghimi, J., 1996. Physics of Fluids, 8 (3), pp. 650-659.

[13] Roth, N., Karl, A., and Frohn, A., 1998. In Proc. 14th Int. Conf. on Liquid Atomization and Spray Systems, Vol. 14, pp. 103-107.

[14] Frohn, A., and Roth, N., 2000. Dynamics of Droplets.

[15] Young, T., 1805. Philosophical Transactions of the Royal Society of London, 95 , pp. 6587.

[16] Marmur, A., Della Volpe, C., Siboni, S., Amirfazli, A., and Drelich, J. W., 2017. Surface Innovations, 5 (1), pp. 3-8.

[17] Fridman, A., 2008. Plasma Chemistry.

[18] Hecht, E. V., ed., 1994. Optik, 3. korr. nachdr. ed.

[19] Zhang, H., Zhang, X., Yi, X., He, F., Niu, F., and Hao, P., 2021. Experimental Thermal and Fluid Science, 124 , p. 110369.

[20] Tanner, L. H., 1979. Journal of Physics D: Applied Physics, 12 (9), sep, pp. 1473-1484. 